\title{
Preparation of inert polystyrene latex particles as microRNA delivery vectors by surfactant-free RAFT emulsion polymerisation
}

Cheuk Ka Poon ${ }^{1}$, Owen Tang ${ }^{2}$, Xin-Ming Chen ${ }^{2}$, Binh T. T. Pham ${ }^{1}$, Guillaume Gody, ${ }^{3}$ Carol A. Pollock ${ }^{2}$, Brian S. Hawkett*1 ${ }^{*}$, Sébastien Perrier*1,3,4

${ }^{1}$ Key Centre for Polymers \& Colloids, School of Chemistry, Building F11, The University of Sydney, NSW 2006, Australia

${ }^{2}$ Kolling Institute of Medical Research, Royal North Shore Hospital and The University of Sydney, St Leonards, NSW 2065, Australia

${ }^{3}$ Department of Chemistry, The University of Warwick, Coventry, CV4 7AL, UK

${ }^{4}$ Faculty of Pharmacy and Pharmaceutical Sciences, Monash University, 381 Royal Parade, Parkville, VIC 3052, Australia

Brian.hawkett@sydney.edu.au; s.perrier@warwick.ac.uk

\section{Supporting information}




\section{Characterisation of PABTC-PAAm macro-RAFT agent}

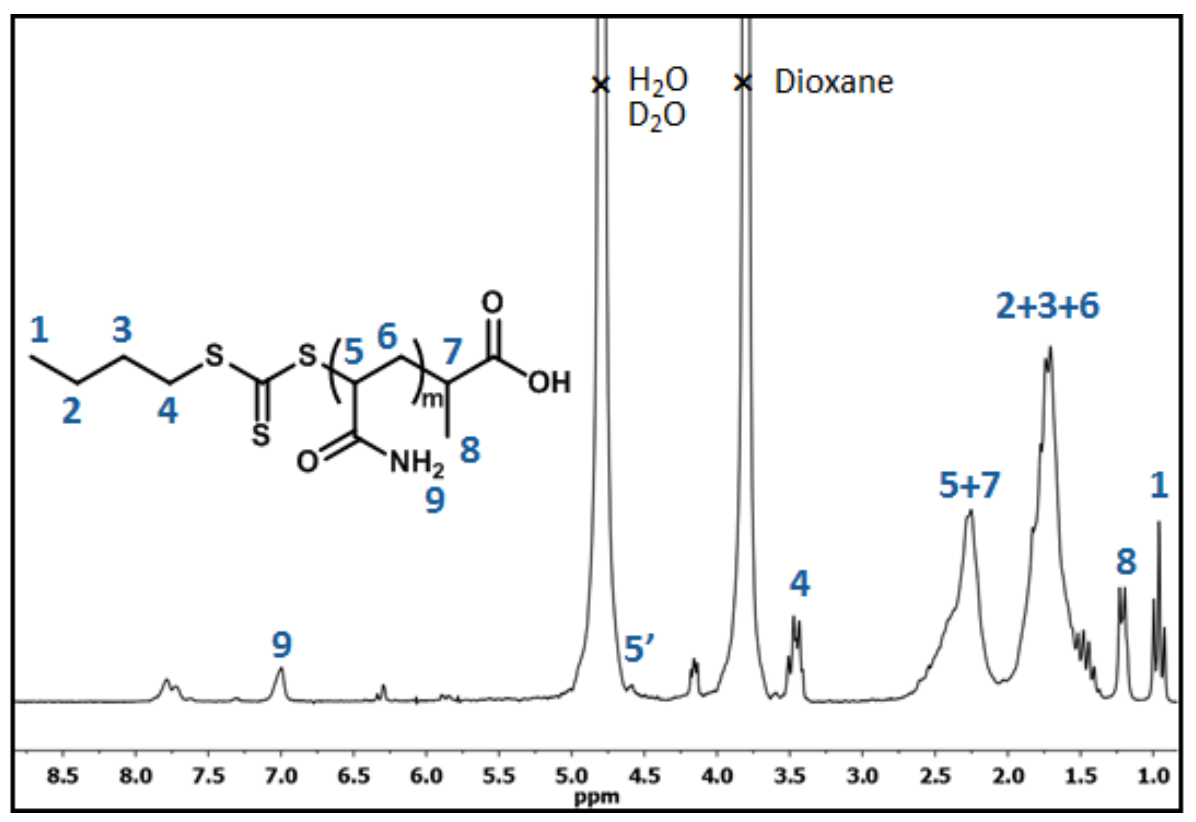

Figure S1. ${ }^{1} \mathrm{H}-\mathrm{NMR}\left(\mathrm{D}_{2} \mathrm{O}\right)$ spectrum of crude HOOC-PAAm macro-RAFT agent (signal 5' corresponds to the proton of the last AAm unit next to the trithiocarbonate group). 


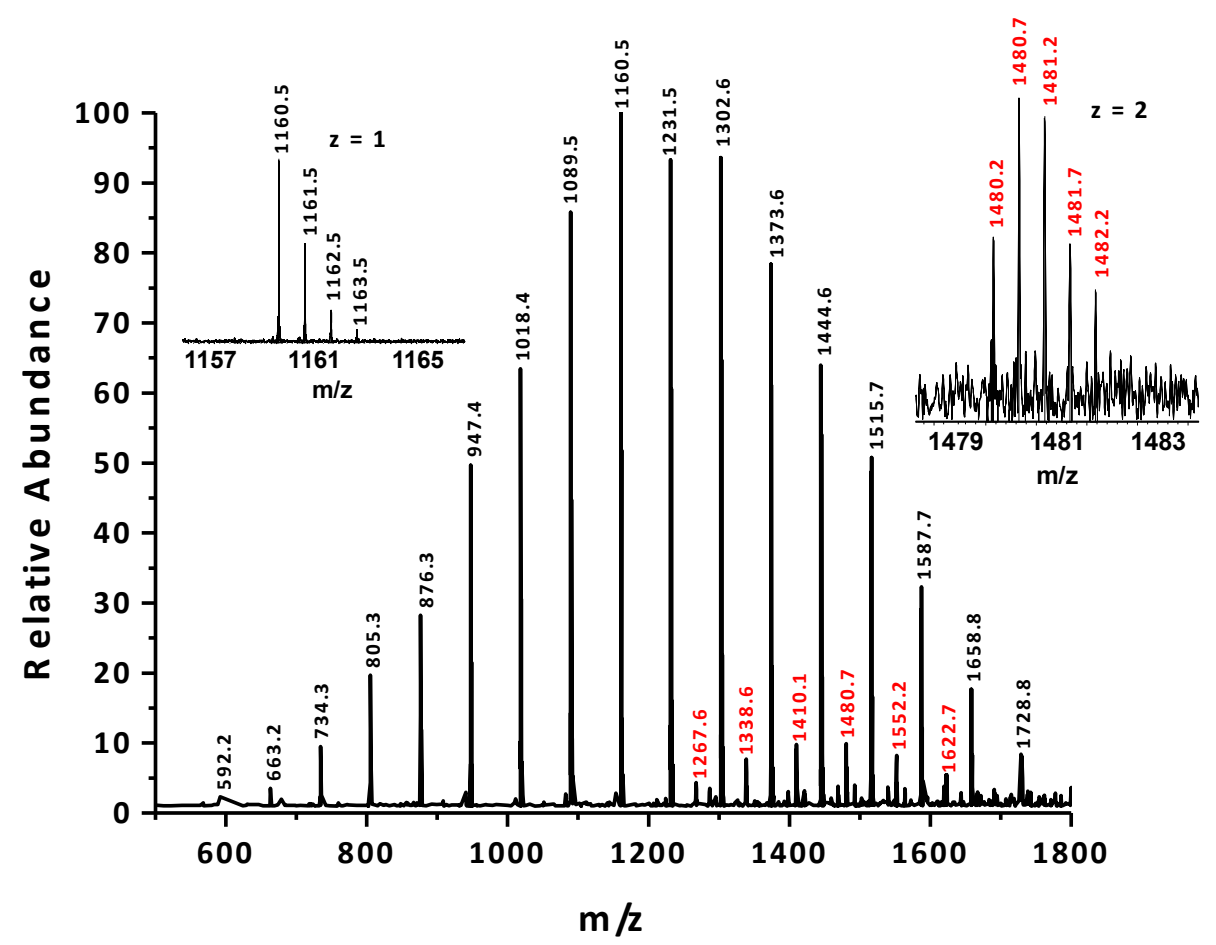

Figure S2. ESI-MS of HOOC-PAAm macro-RAFT agent synthesised from PABTC RAFT agent as described in experimental part and showing two distributions composed by singly(corresponding peaks labelled in black) and doubly-charged species (corresponding peaks labelled in red). Inset figures showing the isotopic peaks of a singly-charged species at $\mathrm{m} / \mathrm{z}$ $=1160.5$ (left) and doubly-charged species at $\mathrm{m} / \mathrm{z}=1480.7$ (right).<smiles>CC(CCC(=O)[O-])CC(C)CC(C)CC(C)CC(C)C(N)=O</smiles>

Figure S3. Example chemical structure of non-living PAAm chains estimated from the minor distribution on ESI-MS (Figure S2). 


\section{Calculations for the livingness of HOOC-PAAm macro-RAFT agent}

Based on the experimental polymerisation conditions used to prepare the macro-RAFT agent HOOC-PAAm, the theoretical fraction of living chains can be estimated and will inform us on the further block extension viability. The livingness (L) can be calculated from Equation 1 below:

$$
L(\%)=\frac{[C T A]_{0}}{[C T A]_{0}+2 \times f \times[I]_{0} \times\left(1-e^{-k_{d} t}\right) \times\left(1-\frac{f_{C}}{2}\right)} \times 100
$$

\section{Equation 1}

where $[C T A]_{0}$ and $[I]_{0}$ correspond to the initial concentrations of PABTC and V-501 azoinitiator, respectively. The whole number ' 2 ' denotes the number of primary radicals resulted from the decomposition of one azoinitiator molecule; ' $f$ ' defines the initiation efficiency of the primary radicals ( $f$ considered to be around 0.5 for $\mathrm{V}-501$ ); while ' $f_{c}$ ', the coupling factor, represents the fraction of chains generated from bimolecular termination events. Based on the doubly-charged species observed after ESI-MS characterisation of the HOOC-PAAm macro-RAFT agent (see Figure S2), a $f_{c}$ value of 1 (i.e. $100 \%$ termination by combination) was considered in this study. ${ }^{24,38-40}$ The decomposition rate coefficient of azoinitiator $\left(k_{d}\right)$ is dependent on the polymerisation solvent and temperature. It can be found or estimated from data in the literature. ${ }^{1}$

Since a binary solvent (dioxane/water, 60:40 v/v) was used in the current system to polymerise AAm, the $k_{\mathrm{d}}$ values of $\mathrm{V}-501$ in $100 \%$ dioxane $\left(3.76 \times 10^{-5} \mathrm{~s}^{-1}\right)$ and $100 \%$ water $\left(2.22 \times 10^{-5} \mathrm{~s}^{-1}\right)$ at $70{ }^{\circ} \mathrm{C}$ were considered to estimate the polymer livingness. ${ }^{2,3}$ 
Substituting the corresponding values into Equation 1 yielded $97.4 \%$ and $98.2 \%$ livingness for $100 \%$ dioxane and $100 \%$ water, respectively. It can be concluded that between $97.4 \%$ and $98.3 \%$ of the PAAm chains carry a thiocarbonylthio moiety and that the same percentage of chains also possess the $\mathrm{COOH}$ functional $\alpha$ chain-end. Based on those theoretical calculation, the final percentage of chains having both $\mathrm{COOH}$ and the thiocarbonylthio moiety can be estimated between $94.9 \%$ and $96.5 \%$. With only a small percentage of non-living chains present (i.e. between $1.7 \%$ and $2.6 \%$ ), as well as high AAm monomer conversion, HOOC-PAAm macro-RAFT agent can be directly used for the next block extension with styrene.

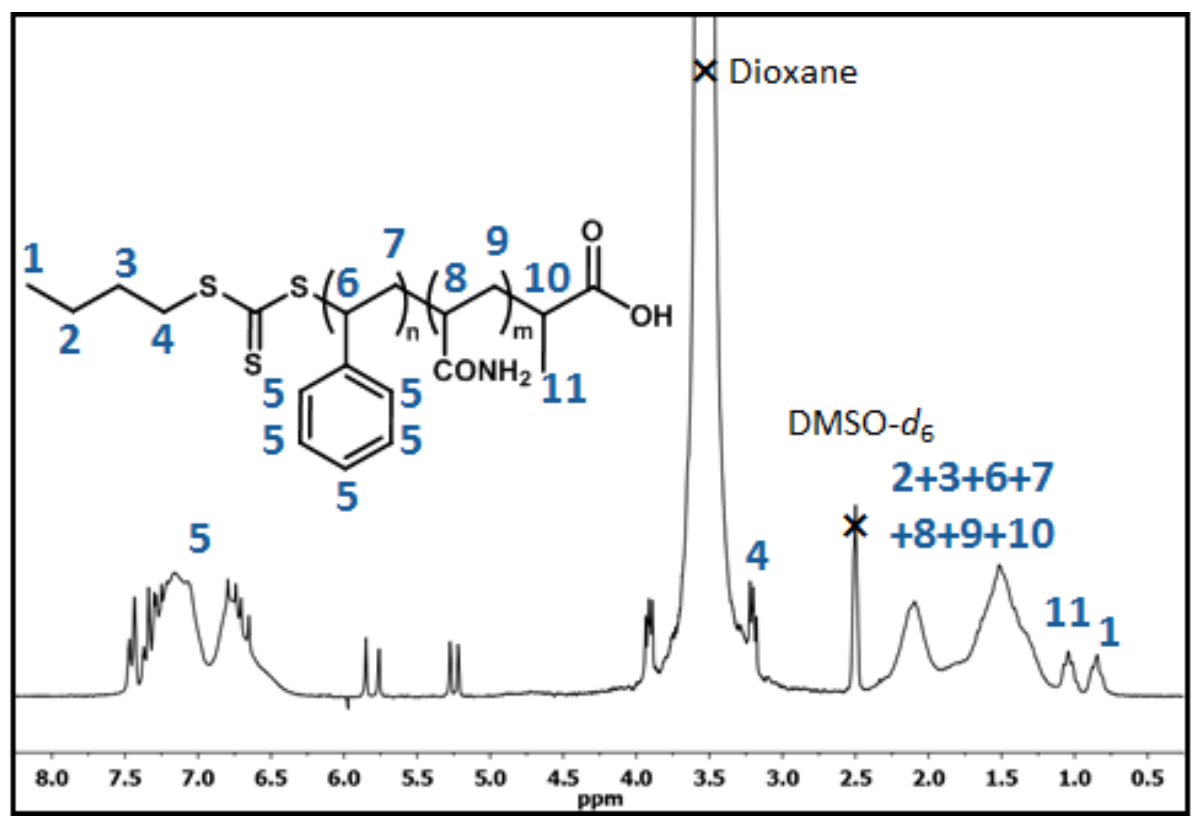

Figure S4. ${ }^{1} \mathrm{H}-\mathrm{NMR}\left(\mathrm{DMSO}-d_{6}\right)$ spectrum of crude HOOC-PAAm- $b$-PS. 


\section{Surface coverage of PS latex particles}

The surface area of particle core covered by each copolymer $\left(A_{\text {macro-RAFT }}\right)$ could be calculated from Equation 2.

$$
A_{\text {macro-RAFT }}=\frac{\pi D_{n, T E M}{ }^{2}}{N_{\text {macro-RAFT per particle }}}
$$

Equation 2

where $D_{n, T E M}$ defines the particle core diameter $(\mathrm{cm})$ measured from TEM; $N_{\text {macro-RAFT per particle }}$ represents the number of copolymers (stabilisers) on each particle. $N_{\text {macro-RAFT per particle }}$ from Equation 2 could be obtained by knowing the number of particles $\left(N_{p}\right)$ per litre (L) water phase based on Equation 3 and Equation 4 below.

$$
N_{\text {macro-RAFT per particle }}=\frac{[\text { macro }- \text { RAFT }] N_{A}}{N_{p}}
$$

Equation 3

$$
N_{p}=\frac{6 \tau}{\pi D_{n, T E M}{ }^{3} d_{p}}
$$

Equation 4

where $N_{A}$ denotes the Avogadro's constant; $\tau$ corresponds to latex particle solids content $\left(\mathrm{g} \cdot \mathrm{L}^{-1}\right)$ and $d_{p}$ represents PS density $\left(\mathrm{g} \cdot \mathrm{mL}^{-1}\right)$ at emulsion polymerisation temperature $\left(d_{p}\right.$ 
$=1.045 \mathrm{~g} \cdot \mathrm{mL}^{-1}$ for PS at $70{ }^{\circ} \mathrm{C}^{4}$ was considered for the current studies) ${ }^{5,6}$ The calculated $N_{\mathrm{p}}, N_{\text {macro-RAFT per particle }}$ and $\mathrm{A}_{\text {macro-RAFT }}$ values for the latex particles synthesised are summarised in Table S1 below.

Table S1. Stability studies of PS latex particles.

\begin{tabular}{ccccc}
\hline $\begin{array}{c}D_{\mathbf{n}, \text { TEM }} \\
(\mathbf{n m})\end{array}$ & $\begin{array}{c}\boldsymbol{\tau} \\
\left(\mathrm{g} \cdot \mathrm{L}^{-1}\right)\end{array}$ & $\begin{array}{c}\boldsymbol{N}_{\mathbf{p}} \times \mathbf{1 0}^{\mathbf{1 8}} \\
\left(\mathbf{L}^{\mathbf{- 1}}\right)\end{array}$ & $\boldsymbol{N}_{\text {macro-RAFT per particle }}$ & $\begin{array}{c}\mathbf{A}_{\text {macro-RAFT }} \\
\left(\mathbf{n m}^{\mathbf{2}}\right)\end{array}$ \\
\hline 7.5 & 3.7 & 16 & 113 & 1.6 \\
\hline
\end{tabular}


${ }^{1}$ H-NMR characterisation of 2-(2-pyridyldithio)ethylamine (PDE) linker

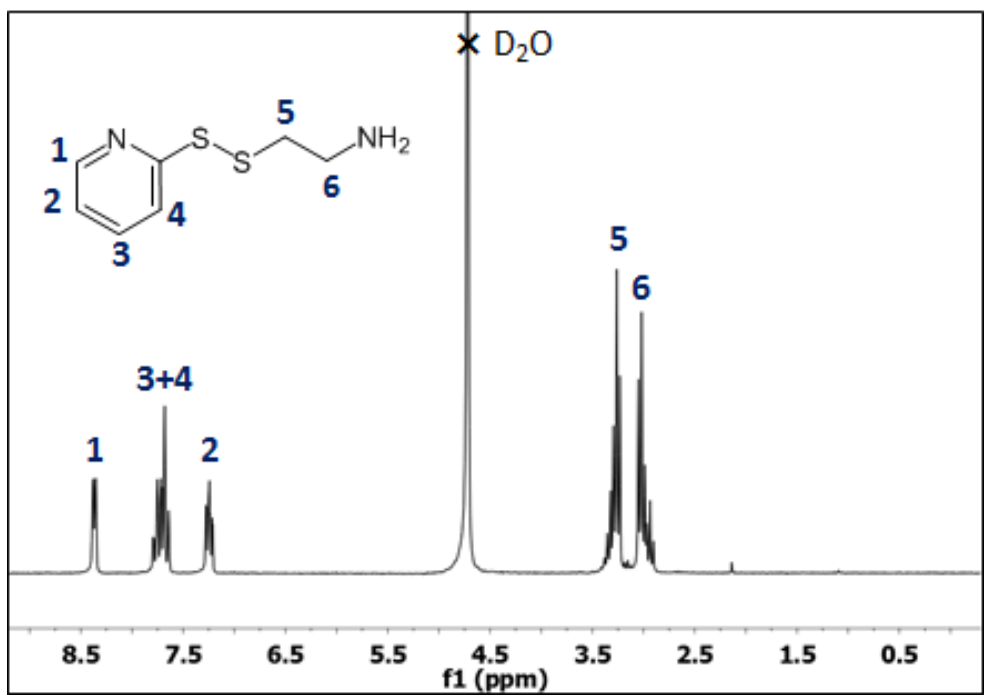

Figure S5. ${ }^{1} \mathrm{H}-\mathrm{NMR}\left(\mathrm{D}_{2} \mathrm{O}\right)$ spectrum of the purified PDE linker.

\section{${ }^{1} \mathrm{H}-\mathrm{NMR}$ characterisation of pyridinethione standard}

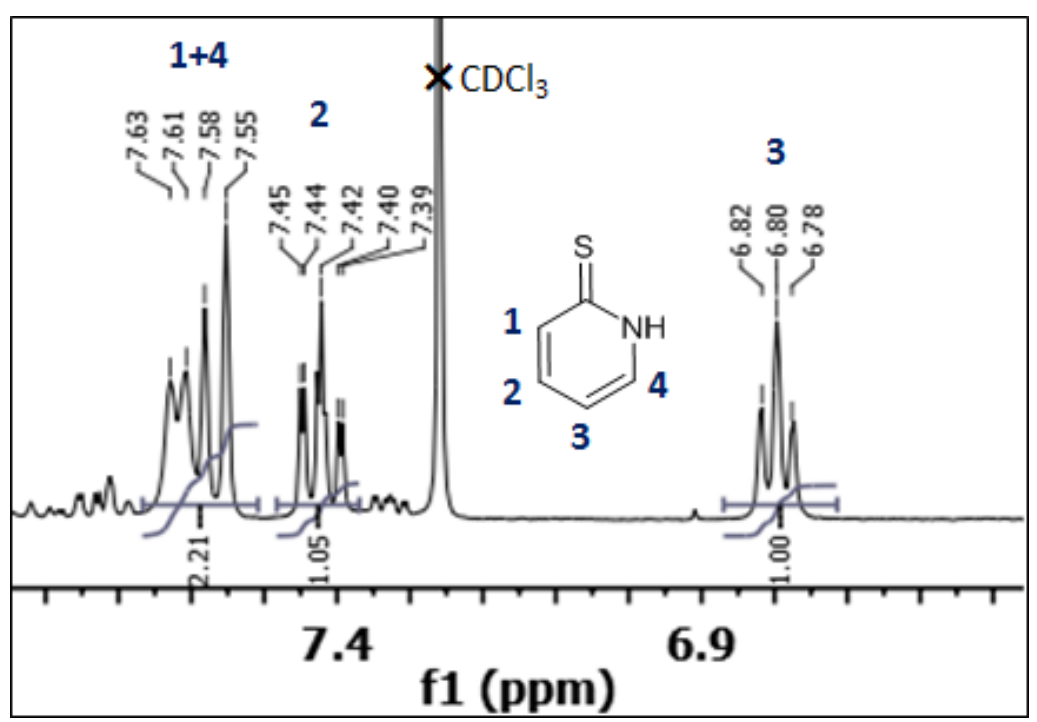

Figure S6. ${ }^{1} \mathrm{H}-\mathrm{NMR}\left(\mathrm{CDCl}_{3}\right)$ spectrum of the purified pyridinethione. 


\subsection{References}

1. P. Nesvadba, in Encyclopedia of Radicals in Chemistry, Biology and Materials, John Wiley \& Sons, Ltd, 2012.

2. S. P. Vernekar, N. D. Ghatge and P. P. Wadgaoknar, J. Polym. Sci. A Polym. Chem., 1988, 26, 953-958.

3. G. Gody, T. Maschmeyer, P. B. Zetterlund and S. Perrier, Macromolecules, 2014, 47, 639-649.

4. D. E. Ganeva, E. Sprong, H. de Bruyn, G. G. Warr, C. H. Such and B. S. Hawkett, Macromolecules, 2007, 40, 6181-6189.

5. C. J. Ferguson, R. J. Hughes, D. Nguyen, B. T. T. Pham, R. G. Gilbert, A. K. Serelis, C. H. Such and B. S. Hawkett, Macromolecules, 2005, 38, 2191-2204.

6. M. Chenal, L. Bouteiller and J. Rieger, Polym. Chem., 2013, 4, 752-762. 